\title{
A COMMENT ON THE STEADY-STATE PERFORMANCE OF MULTISTATE MONOTONE SYSTEMS
}

\author{
BENT NATVIG, ${ }^{*}$ University of Oslo
}

In Iyer (1988) the steady-state expected system state, $\bar{E} \phi(X)$, is derived for a multistate coherent system for the case where each component changes states in time according to a semi-Markov process. These stochastic processes are assumed to be mutually independent. This steady-state expected system state is obviously obtained by the formula

$$
\bar{E} \phi(X)=\sum_{j=1}^{M} \bar{P}(\phi(X) \geqq j),
$$

where $\bar{P}(\phi(X) \geqq j)$ is the steady-state system availability to level $j$. Level $M$ corresponds to the perfect functioning state, whereas level 0 corresponds to complete failure.

From (1) above and by applying the first equation of (4.4) of Natvig and Streller (1984) both on system and component level the main result (2) of Iyer (1988) follows immediately without any semi-Markov process assumption. In Natvig and Streller (1984) the steady-state behaviour of multistate monotone systems is considered by applying the theory for stationary and synchronous processes with an imbedded point process. General results on the steady-state availabilities, steady-state interval availabilities and steady-state mean interval performance probabilities are given. The case of mutually independent component performance processes, as considered in Iyer (1988), is treated specifically.

\section{References}

IYER, S. (1988) Asymptotic performance of a multistate coherent system. Adv. Appl. Prob. 20, 241-243.

NATVIG, B. AND STREller, A. (1984) The steady-state behaviour of multistate monotone systems. J. Appl. Prob. 21, 826-835.

Received 2 August 1988; revision received 5 December 1988.

* Postal address: Institute of Mathematics, University of Oslo, P.O.B. 1053, Blindern, 0316 Oslo 3, Norway. 